\title{
Could patients benefit from whole-brain radiotherapy with unilateral hippocampus sparing?
}

\author{
Lucas Gomes Sapienza ${ }^{1}$, Michelle S. Ludwig ${ }^{1}$, Jacob J. Mandel ${ }^{2}$, Dung H. Nguyen BS ${ }^{1}$, \\ Alfredo Enrique Echeverria ${ }^{1}$ \\ ${ }^{1}$ Department of Radiation Oncology, Dan L. Duncan Comprehensive Cancer Center, Baylor College of Medicine, Houston, Texas, United States \\ ${ }^{2}$ Department of Neurology, Dan L Duncan Comprehensive Cancer Center, Baylor College of Medicine, Houston, Texas, United States
}

Key words: radiotherapy; brain metastasis; hippocampus; neuro-cognitive function

Rep Pract Oncol Radiother 2021;26(3):454-456

Evidence that the hippocampus plays an important role in advanced neuro-cognitive functions (NCF), including the formation of new memories, has accumulated since the 1950s [1]. Reports describing bilateral lesions in the hippocampus that developed clinically detectable neurological impairments suggest that bilateral damage of this structure have negative effects [2-3]. Recently, the randomized NRG-CC001 study [4] provided additional evidence that this structure is related to the memory functions of the brain. The authors found that in the setting of whole brain irradiation (WBRT) with memantine, lowering the bilateral hippocampal radiation dose preserved NCF and patient-reported symptoms. Importantly, relapses in the hippocampus avoidance (HA) region did not increase - occurring in 11 of 257 patients in the HA-WBRT plus memantine arm versus 16 of 261 in the WBRT plus memantine arm.

In both scenarios, bilateral hippocampus alteration (damage or protection) resulted in detectable effect on NCF (impairment or preservation, respectively). An intriguing subsequent question that arises is if the integrity of both hippocampi is a necessary condition for normal - or partially normal - NCF.
The implications of this question for the treatment of brain metastases with irradiation are numerous. If the hippocampus works as a serial organ (similar to spinal cord), bilateral hippocampal sparing would be necessary to preserve function. On the other hand, if the hippocampus behaves as a parallel organ (similar to the lung or the motor cortex of the brain), the avoidance of one side could result in at least partial neuro-cognitive benefit. The latter scenario would expand the indications for hippocampus avoidance because it would include patients with unilateral hippocampus metastasis that are generally not considered candidates for hippocampal preservation. In the third possibility, in which only one specific side is responsible for the function based on dominance $[5,6]$ (e.g. the Broca's area responsible for the speech production), the hippocampal sparing could be unilateral instead of bilateral. In order to generate clinical data to help answer this question, it is important to routinely document dose to each hippocampi - including incidental dose from focal treatments [7] — and to determine dominant hemisphere for each particular patient.

Unilateral avoidance is technically feasible with modern radiotherapy modalities [8-9]. As an illustrative example, Figure 1A-D presents WBRT plans with 


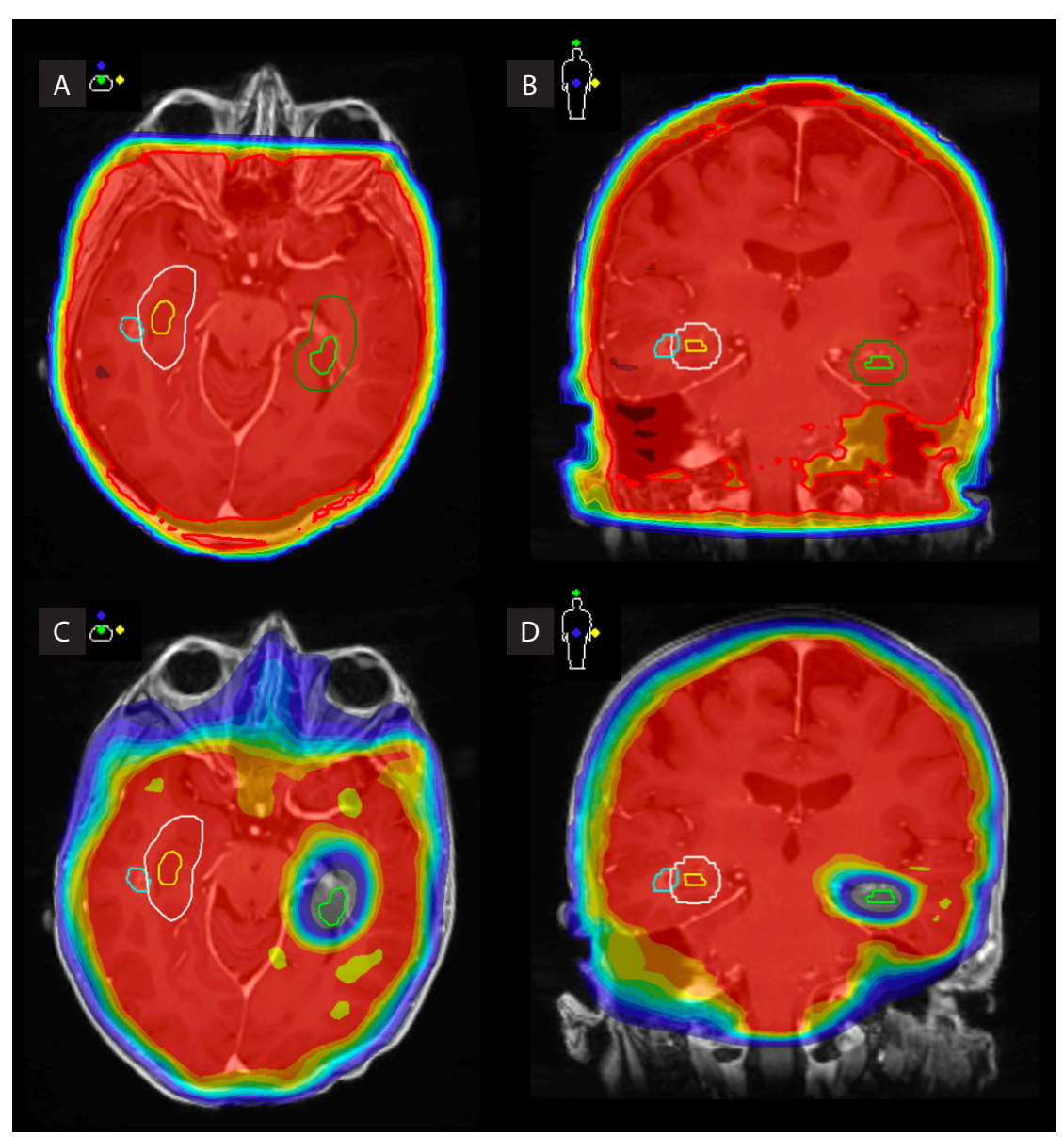

Figure 1. Axial and coronal views of 3D conformal WBRT plan without hippocampus sparing (A, B) and volumetric modulated arc therapy (VMAT) plan with unilateral hippocampus sparing (C, D). Red: 30 Gy isodose. Dark blue: 15 Gy isodose. Cyan: right temporal lobe metastasis. Yellow: right hippocampus. White: right hippocampus $+5 \mathrm{~mm}$. Light green: left hippocampus. Dark Green: left hippocampus $+5 \mathrm{~mm}$. Left hippocampus $\mathrm{D}_{100 \%} 8.5 \mathrm{~Gy}$ (target $\leq 9 \mathrm{~Gy}$ ) and $\mathrm{D}_{\max } 14.4 \mathrm{~Gy}$ (target $\leq 16 \mathrm{~Gy}$ )

and without hippocampus sparing for a 62 years-old female with multiple brain metastases (eight) from high-grade serous carcinoma of the ovary. After reviewing the thin slice MRI ( $1 \mathrm{~mm}-1 \mathrm{~mm})$, one lesion was close to the right hippocampus, but no lesions were near the left hippocampus. If WBRT is indicated, the lesion near the right hippocampus would need to be included. However, could the left hippocampus be avoided? Sparing of the left side could potentially preserve NCF if the hippocampus is considered a parallel organ or if the left side is dominant.

The exact working mechanism of the hippocampus is an intriguing and motivating area of translational research. Further investigation is necessary in determining the impacts of radiation on this bilateral organ, and whether patients with unilateral involvement of the hippocampus may be considered candidates for contralateral preservation.

\section{Conflicts of interest}

None declared.

\section{Funding}

None declared.

\section{Acknowledgments}

None declared.

\section{References}

1. Scoville WB, Milner B. Loss of recent memory after bilateral hippocampal lesions. J Neurol Neurosurg Psychiatry. 1957; 20(1): 11-21, doi: 10.1136/jnnp.20.1.11, indexed in Pubmed: 13406589.

2. Di Gennaro G, Grammaldo LG, Quarato PP, et al. Severe amnesia following bilateral medial temporal lobe damage occurring on two distinct occasions. Neurol Sci. 2006; 27(2): 129-133, doi: 10.1007/s10072-006-0614-y, indexed in Pubmed: 16816912. 
3. Zola-Morgan S, Squire LR, Amaral DG. Human amnesia and the medial temporal region: enduring memory impairment following a bilateral lesion limited to field CA1 of the hippocampus. J Neurosci. 1986; 6(10): 2950-2967, indexed in Pubmed: 3760943.

4. Brown PD, Gondi V, Pugh S, et al. for NRG Oncology. Hippocampal Avoidance During Whole-Brain Radiotherapy Plus Memantine for Patients With Brain Metastases: Phase IIITrial NRG Oncology CC001.J Clin Oncol. 2020; 38(10): 1019-1029, doi: 10.1200/JCO.19.02767, indexed in Pubmed: 32058845.

5. Pospisil P, Kazda T, Hynkova L, et al. Post-WBRT cognitive impairment and hippocampal neuronal depletion measured by in vivo metabolic MR spectroscopy: Results of prospective investigational study. Radiother Oncol. 2017; 122(3):373-379, doi: 10.1016/j.radonc.2016.12.013, indexed in Pubmed: 28063694.

6. Goda JS, Dutta D, Krishna U, et al. Hippocampal radiotherapy dose constraints for predicting long-term neurocognitive outcomes: mature data from a prospective trial in young patients with brain tumors. Neuro Oncol. 2020; 22(11): 1677-1685, doi: 10.1093/neuonc/noaa076, indexed in Pubmed: 32227185.

7. Fiorentino A, Tebano U, Sicignano G, et al. Hippocampal dose during Linac-based stereotactic radiotherapy for brain metastases: An observational study. Phys Med. 2018; 49: 135-138, doi: 10.1016/j.ejmp.2017.09.129, indexed in Pubmed: 28951047.

8. Giaj Levra N, Sicignano G, Fiorentino A, et al. Whole brain radiotherapy with hippocampal avoidance and simultaneous integrated boost for brain metastases: a dosimetric volumetric-modulated arc therapy study. Radiol Med. 2016; 121(1): 60-69, doi: 10.1007/s11547-015-0563-8, indexed in Pubmed: 26231251.

9. Kazda T, Vrzal M, Prochazka T, et al. Left hippocampus sparing whole brain radiotherapy (WBRT): A planning study. Biomed Pap Med Fac Univ Palacky Olomouc Czech Repub. 2017; 161(4): 397-402, doi: 10.5507/bp.2017.031, indexed in Pubmed: 28731074. 